\title{
La calidad de vida como concepto
}

\author{
Luz Marina Bautista-Rodríguez
}

ISSN 1794-9831

E-ISSN 2322-7028

Vol. 14 No. 1

Ene - Jun 2017

Cúcuta, Colombia

Fue ya a finales de los años 60 y en la década de los 70 cuando el concepto de calidad de vida se vinculó a una noción que incorporaba medidas subjetivas, es decir, evaluaciones que incluían aspectos como los sentimientos personales de felicidad o satisfacción. A partir de entonces hubo una evolución muy significativa del concepto de calidad de vida que implicó pasar de entender el bienestar en términos objetivos a incluir la perspectiva subjetiva de satisfacción con la vida. Este nuevo perfil le dio una entidad propia, diferenciándolo de los indicadores sociales y acentuando la percepción del sujeto sobre su vida y sobre las condiciones objetivas de su existencia.

García (4) menciona que "desde los años 80 se ha venido desarrollando una consolidación del concepto de calidad de vida. Los avances de los últimos tiempos han conseguido incrementar la comprensión del concepto, su importancia y su uso, aunque algunos aspectos del constructo calidad de vida se encuentran en su etapa infantil y hoy siguen siendo motivo de debate".

De otra parte, la calidad de vida es un concepto complejo que refleja aspectos sociales e individuales -objetivos y subjetivos- y múltiples ámbitos como son el físico, el emocional, el educativo y el laboral. Desde diversas disciplinas se ha abordado también el concepto de calidad de vida. Así, en medicina tuvo un importante significado en el siglo pasado debido al incremento de enfermedades crónicas y a la posibilidad del diagnóstico temprano de las mismas y su posterior tratamiento, lo que suponía prolongar la vida de los pacientes que antes tendrían dificultades para sobrevivir. Comenzó a plantearse la idea no sólo de cantidad sino de calidad de los años ganados a partir de las anteriores intervenciones (4).

García (4) destaca que "la psicología asumió el concepto de calidad de vida y le abrió un campo de investigación fundamental, el bienestar percibido. Múltiples ramas de la psicología como la personalidad, social, del desarrollo o cognitiva aportaron elementos

\footnotetext{
* Enfermera. Magister en Enfermería con Énfasis en Gerencia en Servicios en Salud. Docente. Universidad Francisco de Paula Santander. Cúcuta, Colombia.

Correo electrónico: luzmarinabr@ ufps.edu.co
} 
E-ISSN 2322-7028

Vol. 14 No. 1

Ene - Jun 2017

Cúcuta, Colombia de interés en la explicación de vivencias de satisfacción o insatisfacción con las experiencias vitales. Alguno lo llamó, incluso, psicología hedonista... Desde los planteamientos económicos, políticos y sociales, hablar de calidad de vida ha sido muy útil puesto que supone hablar de cambios y efectos de las políticas sociales y de eficacia en los servicios" (4).

Vinaccia y Orozco (5) resaltan que hacia mediados de los años 50 se incluyó el concepto de calidad de vida en el área de la medicina y luego se asumió en la psicología. Desde ese momento hasta hoy, el concepto ha experimentado varias modificaciones. Inicialmente se refería al cuidado de la salud personal, luego pasó a ser la preocupación por la salud e higiene pública. Posteriormente, hizo alusión a los derechos humanos, laborales y ciudadanos; después, a la capacidad de acceso a los bienes económicos y, finalmente, se convirtió en la preocupación por la experiencia del sujeto de su vida social, de su actividad cotidiana y de su propia salud. El concepto está de moda $\mathrm{y}$, como muchos otros términos, es polivalente, multidimensional y se usa con significados muy diferentes.

Para Puig (6) "la satisfacción de las necesidades es un elemento clave en la percepción de la calidad de vida. A lo largo de la historia, distintas disciplinas han intentado explicar las causas de la felicidad y la calidad de vida". Además, señala que "en la época preindustrial se hablaba sobre todo de felicidad, valorando la espiritualidad y subjetividad; en la etapa industrial se hablaba de bienestar, relacionando el término con el materialismo y el utilitarismo, y es apenas en la etapa postindustrial cuando se empieza a conceptualizar el constructo calidad de vida, que aparece como resultado de los rápidos y continuos cambios sociales" (6).

Con el paso del tiempo, distintos autores y organizaciones han intentado conceptualizar la calidad de vida desde distintos enfoques. Este constructo ha evolucionado desde una concepción básicamente materialista, en la que primaban los aspectos objetivos de nivel de vida, pasando por la perspectiva donde los aspectos subjetivos se constituyen en el sustento fundamental, hasta la situación actual, en la que se adjudica la subjetividad y el carácter multidimensional del constructo, de forma que, aunque no hay un consenso único, parece que hay un cierto acuerdo en aceptar valorarla de forma multidimensional, identificando componentes objetivos y subjetivos (6).
Gómez y Sabeh (7) en su análisis, han reflejado con claridad el constructo calidad de vida y lo asumen constituido por tres elementos en interacción: 1. Las condiciones objetivas de vida, o descripción objetiva de los individuos y sus circunstancias. 2. El bienestar subjetivo, definido como la satisfacción personal con esas condiciones y con el estilo de vida. 3. Los valores personales y aspiraciones. En este sentido, se señalan dos ámbitos básicos del constructo, que la mayoría de los autores indican: la dimensión objetiva (condiciones de vida experimentadas por las personas) y la dimensión subjetiva (la valoración del individuo sobre distintos aspectos de su existencia) (7).

De otro lado, para Melchiors et al. (8), la calidad de vida es un concepto multidimensional que incluye una diversidad de factores, entre los cuales el estilo de vida, la vivienda, la satisfacción con el trabajo, la situación económica, el acceso a los servicios públicos, las comunicaciones, la urbanización y la criminalidad, entre otros, que la componen en el contexto social y que influencian el desarrollo humano de una comunidad.

Según Melchiors et al. (8), la calidad de vida (CV) abarca el bienestar físico, social, psicológico y espiritual. El bienestar físico se determina por la actividad funcional, la fuerza o la fatiga, el sueño y el reposo, el dolor y otros síntomas. El bienestar social tiene que ver con las funciones y las relaciones, el afecto y la intimidad, la apariencia, el entretenimiento, el aislamiento, el trabajo, la situación económica y el sufrimiento familiar. El bienestar psicológico se relaciona con el temor, la ansiedad, la depresión, la cognición y la angustia que genera la enfermedad y el tratamiento. El bienestar espiritual, por su parte, abarca el significado de la enfermedad, la esperanza, la trascendencia, la incertidumbre, la religiosidad y la fortaleza interior.

De acuerdo con López y Rodríguez (9), la calidad de vida implica "la evaluación global que un sujeto hace de su vida, con base en sus características personales (demográficas, valores, personalidad) y en factores externos entre los que se encuentran las enfermedades y el tratamiento que éstas requieren".

La Organización Mundial de la Salud (OMS) define la calidad de vida como "la percepción del individuo de su posición en la vida en el contexto de la cultura y sistema de valores en los que vive y en relación con sus objetivos, expectativas, estándares y preocupaciones" $(6,10)$. Se trata de un concepto muy amplio que está influido de modo complejo por 
la salud física del sujeto, su estado psicológico, su

nivel de independencia, sus relaciones sociales, así

Vol. 14 No. 1

como su relación con los elementos esenciales de

Ene - Jun 2017

su entorno. De este modo, la calidad de vida es un

Cúcuta, Colombia

constructo multidimensional que comprendería tres

dimensiones fundamentalmente (10):

- Dimensión física: percepción del estado físico o la salud, entendida como ausencia de enfermedad, los síntomas producidos por la enfermedad, y los efectos adversos del tratamiento.

- Dimensión psicológica: percepción del individuo sobre su estado cognitivo y afectivo como el miedo, la ansiedad, la incomunicación, la pérdida de autoestima, la incertidumbre del futuro, etc. También incluye tanto las creencias personales, espirituales y religiosas como el significado de la vida y la actitud ante el sufrimiento.

- Dimensión social: percepción del individuo sobre las relaciones interpersonales y los roles sociales en la vida como la necesidad de apoyo familiar y social, la relación médico-paciente y el desempeño laboral. 


\section{REFERENCIAS BIBLIOGRÁFICAS}

Ene - Jun 2017

1. Moreno-Jiménez B, Ximénez-Gómez C. Evaluación de la calidad de vida. Manual de Evaluación en Psicología Clínica y de la Salud. [Internet]. 1996 [consultado 22 de enero de 2010]; p. 1045-1067. Disponible en: https:// www.uam.es/gruposinv/esalud/Articulos/Personalidad/evaluacion-de-calidaddevida.pdf

2. Casas-Aznar F. El concepto de calidad de vida en la intervención social en el ámbito de la infancia. En: III Jornadas de Psicología de la Intervención Social. Madrid: Ministerio de Asuntos Sociales; 1991. p. 649-672.

3. Lindström B. The essence of existence. On the quality of life of children in the Nordic Countries. Theory and practice in public health. [Tesis doctoral]. Göteborg: The Nordic School of Public Health; 1994.

4. García-Gómez JL. Evaluación de la calidad de vida en los pacientes con diabetes mellitus tipo 1: el caso del Hospital Universitario nuestra Señora de candelaria de Tenerife. [Tesis doctoral]. Tenerife, España: Universidad de la Laguna; 2005. p. 681.

5. Vinaccia S, Orozco LM. Aspectos psicosociales asociados con la calidad de vida de las personas con enfermedades crónicas. Perspectivas en Psicología 2005; 1(2): 125-137.

6. Puig-Obet M. Cuidados y calidad de vida en Vilafranca del Penedès: los mayores de 75 y más años atendidos por el servicio de atención domiciliaria y sus cuidadores familiares. [Tesis doctoral en Internet]. Barcelona: Universidad de Barcelona; 2009 [consultado 8 de enero de 2011]; p. 364. Disponible en: http://diposit.ub.edu/ dspace/handle $/ 2445 / 43013$ ?mode $=$ full

7. Gómez M, Sabeh EB. Calidad de vida. Evolución del concepto y su influencia y la práctica España. Instituto Universitario de Integración en la Comunidad, Facultad de Psicología, Universidad de Salamanca. [Internet]. 2000 [consultado 20 de enero de 2010]. Disponible en: http://campus.usal.es/ inico/investigacion/invesinico/ calidad.htm

8. Melchiors AC, Correr CJ, Rossignoli P, Pontarolo R, Fernández F. Medidas de evaluación de la calidad de vida en Diabetes. Parte I: Conceptos y criterios de revisión. Seguim Farmacoter 2004; 2(1): 1-11.

9. López-Carmona JM, Rodríguez-Moctezuma R. Adaptación y validación del instrumento de calidad de vida Diabetes 39 en pacientes mexicanos con diabetes mellitus tipo 2. Salud Pública de México 2006; 48(3): 200-211.

10. Machado-Romero A, Anarte-Ortíz MT, Ruiz de Adana-Nava MS. Predictores de calidad de vida en pacientes con diabetes mellitus Tipo 1. Clínica y Salud [Internet]. 2010 [consultado 16 de julio de 2016]; 21(1): 35-47. Disponible en: http://www.redalyc.org/articulo.oa?id=180615542004 